\title{
La educación en contextos de diversidad cultural: un estudio sobre educadores brasileños
}

\author{
Education in contexts of cultural diversity: a study on brazilian educators \\ Educação em contextos da diversidade cultural: um estudo sobre \\ educadores brasileiros
}

\author{
Valentín Martínez-Otero Pérez \\ Universidad Complutense de Madrid (UCM), España
}

\begin{abstract}
Resumen
Este artículo se interesa por la educación en contextos de diversidad cultural y por la perspectiva de los educadores sobre sus necesidades formativas. Se utilizó un cuestionario diseñado ad hoc y se opta por un método mixto, cuantitativo y cualitativo, mediante un diseño complementario de fases paralelas (diseño anidado) Cuan-cual. El cuestionario se aplicó a una muestra de 237 educadores brasileños. Los resultados revelan que la atención a la diversidad cultural ocupa un papel importante en la actividad educativa. Los educadores, en su conjunto, son sensibles a la diversidad cultural y a su fomento, pero la formación intercultural recibida es insuficiente. Se fomenta poco la participación familiar, los recursos escasean y la dimensión intercultural carece de sistematización pedagógica.
\end{abstract}

Palabras clave: diversidad cultural; interculturalidad; formación; profesionales de la educación.

\section{Resumo}

Este artigo foca-se na educação em contextos de diversidade cultural e pela perspectiva dos educadores sobre suas necessidades de formação. É utilizado um questionário elaborado ad hoc, e escolhe-se um método misto, quantitativo e qualitativo, por meio de um design complementar de fases paralelas (design aninhado) Quan-cual. O questionário foi aplicado a uma amostra de 237 educadores brasileiros. Os resultados revelam que a atenção à diversidade cultural desempenha um papel importante na atividade educacional. Os educadores, em conjunto, são sensiveis à diversidade cultural e à sua promoção; no entanto, a formação intercultural recebida é insuficiente. A participação das famílias é pouco incentivada, os recursos são escassos e a dimensão intercultural carece de sistematização pedagógica.

Palavras-chave: diversidade cultural; interculturalidade; formação; profissionais da educação.

\begin{abstract}
This article is interested in education in contexts of cultural diversity and the educators' perspective on their training needs. A questionnaire designed ad hoc was used and a mixed method is chosen, quantitative and qualitative, through a complementary design of parallel phases (nested design) Quan-qual. which through a complementary design of parallel phases (nested design). The questionnaire was applied to a sample of 237 Brazilian educators. The results reveal that attention to cultural diversity plays an important role in educational activity. Educators, as a whole, are sensitive to cultural diversity and its promotion, but the intercultural training received is insufficient. Family participation is little encouraged, resources are scarce and the intercultural dimension lacks pedagogical systematization.
\end{abstract}

Keywords: cultural diversity; interculturality; training; education professionals.

Como citar (APA):

Martínez-Otero,V. (2021). La educación en contextos de diversidad cultural: un estudio sobre educadores brasileños. Revista Iberoamericana de Educación, 85(2),9-26. https://doi.org/10.35362/ rie8524061 


\section{Introducción}

La diversidad cultural es una realidad escolar y social indiscutible que se halla actualmente en el centro del debate pedagógico. Si partimos de la base de que la sociedad es multicultural el sistema educativo no puede ser homogeneizador.

Defendemos que la estimación de las personas y los grupos de otras culturas es una exigencia de la genuina educación, que se sustenta en la dignidad de todo ser humano, y, por lo mismo, el hecho de que algunos planteamientos teóricos y las prácticas consecuentes, sobre todo de índole asimilacionista, no se atengan a ese elemental principio nos lleva a rechazarlos por inapropiados.

La diversidad cultural plantea retos educativos de carácter escolar y social. ¿En qué medida los educadores perciben que están suficientemente preparados para responder a esos desafíos? Interesa conocer también qué piensan los propios educadores sobre el planteamiento organizativo de las instituciones educativas en que trabajan en lo que se refiere a la atención a la diversidad cultural. Una estancia de investigación nos ha permitido centrarnos en Brasil. Desde luego, este gran país se distingue por la multiculturalidad, por la diversidad étnica y sociocultural de las personas y grupos humanos que lo constituyen. Aunque por su conformación histórica comparte con los otros países de la región el mestizaje como definitorio de su identidad, presenta unos rasgos específicos difíciles de precisar, entre los que se descubre la presencia étnico-cultural africana, europea, indígena y, en menor medida, asiática.

La identidad brasileña no es fácil de delimitar, pues como toda sociedad nos sitúa ante una realidad humana muy variable en su composición, en su forma de vivir, de organizarse y de expresarse, y además en continuo dinamismo. Pese a las dificultades, creemos que la reflexión prudente y el acercamiento investigador a la realidad de los educadores brasileños en contextos escolares y sociales de diversidad son totalmente necesarios, particularmente si se quiere avanzar hacia una convivencia entre personas, comunidades y grupos humanos culturalmente diferenciados, algunos de los cuales, sobre todo los indígenas y afroamericanos, con frecuencia a lo largo de la historia han sido invisibilizados, negados, oprimidos y excluidos. 
El ámbito donde se entrecruzan la pedagogía y la cultura es el que corresponde a la educación intercultural, que asume en este artículo todo el protagonismo, ya sea latente o patente, porque es reflejo de una corriente científica que, con independencia de la pluralidad de ópticas, se ha destacado por su valoración de la diversidad y su compromiso con la inclusión y la convivencia de las personas y grupos étnicos y culturales en contextos escolares y sociales. Entre las variadas perspectivas de educación intercultural cuenta con especial relevancia histórica en Brasil, según defiende Oliveira (2015), la pedagogía de Freire, uno de sus precursores más influyentes, del que debe recordarse, por ejemplo, su insistencia en la trascendencia del respeto a la identidad cultural, de la que forman parte la dimensión individual y de clase de los educandos (Freire, 2019).

\section{Cultura, diversidad cultural y educación}

El concepto de cultura es en sí mismo complejo y no se maneja en el mismo sentido en el ámbito de las ciencias sociales. En ocasiones, remite a la idea de formación de la persona, principalmente en lo que se refiere a los conocimientos, algo que queda reflejado en la expresión "persona culta" y que, con arreglo, a este trabajo sería bastante limitado. En cambio, el acercamiento al significado de la cultura tal como lo manejan sobre todo antropólogos y sociólogos, revela una noción mucho más amplia que nos sitúa ante el conjunto de modos de vida, costumbres, producciones, etc., que se transmiten de una generación a otra y que expresan el grado de desarrollo artístico, científico, industrial, etc., de un grupo social concreto en una determinada época. Desde esta óptica, la cultura, sin soslayar la personalidad, puesto que ambas son interdependientes, tiene un claro componente colectivo que puede acrecentarse o declinar. El término 'cultura', desde una perspectiva antropológica, se aplica a todos los estilos de vida social y es, como afirma Cuche (2012), la que explica que nada sea puramente natural en el ser humano y la que, al mismo tiempo, hace posible la modificación de la naturaleza.

Desde el punto de vista educativo resulta fundamental apreciar y fomentar la cultura y las culturas en cualquiera de sus manifestaciones. Sin cultura, inherente al desarrollo, no puede organizarse una convivencia verdaderamente sólida. Por eso, las raíces culturales han de fortalecerse a nivel individual y social, al tiempo que se enfatiza cuanto contribuye a la convi- 
vencia, que se afirma con el respeto a todas las culturas, conformadoras del rico patrimonio de la humanidad y que no tienen por qué disolverse en beneficio de una cultura única.

La salvaguardia y el cultivo de la diversidad cultural constituyen aspectos irrenunciables en nuestras sociedades $y$, junto a las medidas políticas, jurídicas, laborales, económicas, etc., se precisa un proyecto pedagógico consistente del que la formación de educadores es parte relevante, sobre todo por su efecto irradiador. Al beneficio técnico y humano que una preparación tal reporta a los propios profesionales de la educación se agrega su repercusión sobre los educandos, lo que constituye una auténtica siembra de convivencia.

En Brasil, por ejemplo, país esencialmente multicultural, coexisten e interactúan, no siempre en armonía, una diversidad de grupos étnicos, sociales y culturales, y paralelamente se fortalecen o debilitan desde diferentes ideologías y políticas sociales y educativas las contribuciones de dichos grupos a la construcción de la identidad común. Todo ello sin obviar la considerable mixtura poblacional y cultural existente, una fusión de realidades heterogéneas que no ha disipado, y a veces incluso ha acrecentado, las tensiones, el racismo, la discriminación, etc. Desde sectores críticos se cuestiona, por ejemplo, tal como recogen Oliveira y Candau (2010) al referirse al caso brasileño y tomando como base la propuesta del colectivo latinoamericano Modernidad/Colonialidad, la lógica eurocéntrica dominante y se promueven variadas estrategias desde perspectivas de sectores indígenas y afrodescendientes, tradicionalmente en situación de exclusión, para visibilizar y empoderar su realidad en el marco de un proyecto alternativo al racismo epistémico y a la colonialidad del ser, del saber y del poder. Recientemente, Ferreira y Silva (2020) sostienen que el racismo estructural está bastante presente en el Nordeste brasileño.

Queda mucho por hacer en lo concerniente a la diversidad cultural, a menudo asociada a la desigualdad social que se advierte en la escuela. Beserra y Lavergne (2018) refiriéndose a Brasil señalan que profesores y administradores escolares no han recibido formación suficiente sobre estas cuestiones, al tiempo que agregan que uno de los principales beneficios de celebrar la diversidad es que permite una mayor visibilidad de los grupos sociales más vulnerables. 
Aunque en la sociedad y en los contextos educativos brasileños, como sostiene Candau (2016), hay una creciente sensibilidad hacia la diversidad cultural, la posibilidad de que el profesorado adopte una perspectiva intercultural en sus prácticas cotidianas no es tarea sencilla, pues parece imponerse una política educativa pública homogeneizadora que presiona continuamente a los educadores mediante múltiples mecanismos burocráticos y numerosos instrumentos de evaluación fundamentados en pruebas estandarizadas. Parafraseando a Silva y Rebolo (2017), en los procesos de educación intercultural influyen factores estratégicos, estructurales, tecnológicos, políticos, materiales, pedagógicos, humanos y culturales, cuya desconsideración acrecienta los riesgos de que las acciones educativas resulten estériles. Es evidente que en este marco complejo, junto a las políticas apropiadas y la suficiencia de los recursos, se precisa una adecuada formación de los educadores, muchas veces preparados para trabajar en contextos homogéneos y homogeneizadores.

En el abordaje pedagógico de la diversidad cultural tradicionalmente se han distinguido cuatro modelos, que no son en absoluto monolíticos: asimilacionista, compensatorio, multicultural e intercultural, adoptados por distintos sistemas educativos o por el mismo según los momentos y que, desde perspectivas ideológicas desemejantes a pesar de la evolución que hayan podido experimentar, han repercutido en las acciones educativas y en la sociedad. Grosso modo, los modelos asimilacionista y compensatorio consideran que ciertos grupos humanos, ya sea por motivos de raza, etnia e inmigración, se hallan en situación de inferioridad, incluso deprimidos socioeconómicamente, y con arreglo al discurso del sector hegemónico, advertido en la política educativa, en el diseño curricular, en las prácticas educativas y en los procedimientos de evaluación, destinan a los miembros de los grupos más vulnerables a programas que garanticen su adaptación a la cultura dominante.

El modelo multicultural reconoce la coexistencia de culturas en un mismo espacio o territorio, pero puede que no promueva suficientemente la convivencia entre ellas. Por tanto, uno de los grandes retos que se presentan a las prácticas pedagógicas orientadas desde este modelo es, como apuntan Silva y Vasconcelos (2019), la implantación de mecanismos capaces de garantizar a los distintos grupos culturales el ejercicio pleno de sus derechos fundamentales. Se llega así al modelo intercultural, comprometido con la inclusión, el intercambio constructivo y la igualdad de oportunidades, no solo 
con el reconocimiento de la diversidad cultural, porque, a fin de cuentas, un genuino modelo educativo intercultural, como sugiere Botler (2012), ha de asegurar más democracia.

La educación intercultural, corriente pedagógica extensa, trascendente y con gran desarrollo, no exenta de controversia y compleja en su verificación, no se conforma con la mera yuxtaposición de culturas. Si el multiculturalismo describe una situación de partida, el interculturalismo muestra la dirección que debe seguirse para que la convivencia entre personas y grupos humanos de diferentes culturas en condiciones de influencia paritaria sea fuente de riqueza. Tal como aquí se entiende forma parte de un proyecto pedagógico comprometido con la justicia social. La educación intercultural puede admitir diversas interpretaciones, aunque todas han de cimentarse en la dignidad de la persona.

El hecho de que Brasil y en general el resto de América Latina sean más sensibles a la diversidad cultural, ofrece a priori un marco más favorable para la educación intercultural; sin embargo, la cuestión se torna complicada en la vida cotidiana de las instituciones educativas, en las que permean las históricas y arraigadas desigualdades socioeconómicas de la región. Al respecto, ya indica Senna (2000), que la experiencia brasileña con la educación intercultural revela que la simple aplicación de medidas impulsoras de legitimación y respeto a las identidades culturales en contacto, no son suficientes para avanzar hacia la igualdad social y a veces incluso se traduce en la perpetuación de la brecha social, así como en disputas y conflictos. Para este autor, la aspiración a que la educación intercultural resulte efectiva hace necesarias unas dotaciones del poder público en el marco de una política social genuinamente integradora.

El estudio de Lorente (2019) sobre los problemas que aquejan a los sistemas educativos de la región latinoamericana permite constatar, en general, que las poblaciones afrodescendientes, y más aún los grupos indígenas, están en situación de inferioridad educativa si se les compara con el total de la población. Se corrobora que la desigualdad educativa en función del grupo étnico de pertenencia se agrega a las relativas al nivel socioeconómico del hogar, al área geográfica de residencia o al género. Y todo ello, concluye el estudio, muestra las dificultades de los sistemas educativos latinoamericanos para paliar o revertir las desigualdades de origen. 
Nada justifica la discriminación secular de los pueblos indígenas y afrodescendientes, la exclusión de que son objeto, las negativas condiciones socioeconómicas en que se hallan ni los malos resultados escolares que, en general, obtienen cuando se comparan con los miembros de la cultura dominante.

Igreja (2016), al analizar las diversas acciones que el Estado brasileño ha puesto en práctica durante los últimos años para combatir la discriminación y eliminar las desigualdades históricas de índole racial, destaca los cambios legislativos, el diseño de políticas de acciones afirmativas y la creación de instancias para el recibimiento de quejas y reclamaciones contra actos discriminatorios. A pesar de esa variedad de acciones, la autora subraya que se ha experimentado una considerable frustración por la insuficiente efectividad de estas medidas.

La educación intercultural, que incorpora un discurso inclusivo, es una herramienta fundamental para prevenir y combatir prejuicios sobre los miembros de la comunidad educativa o sus culturas. Por supuesto, se precisa trabajar sistemáticamente la doble vertiente explícita e implícita del currículo, de gran influencia en el proceso educativo y en las relaciones humanas. Se trata de evitar que la escuela, lato sensu, sea caldo de cultivo o correa de transmisión de cualquier forma de discriminación, lo que resultaría totalmente incompatible con su esencia humanista.

Brasil, al igual que toda América Latina, tiene por delante el gran reto de alcanzar una educación de calidad para todos. No escasean los marcos legales y normativos inspirados en declaraciones o convenciones internacionales, pero sigue sin haber una aplicación efectiva, realmente intercultural e inclusiva (Carvalho y Faustino, 2016). En esta coyuntura compleja, nos planteamos un acercamiento a la perspectiva de los educadores sobre sus necesidades formativas en contextos de diversidad cultural.

\section{Método}

Se ha utilizado un cuestionario diseñado ad hoc para conocer la percepción de los educadores sobre necesidades formativas relativas a la diversidad cultural y la inclusión. Se opta por un método mixto, a un tiempo cuantitativo y cualitativo, mediante un diseño complementario de fases paralelas (diseño 
anidado) Cuan-cual. Esta metodología mixta, aunque con predominio de la vertiente cuantitativa, es una excelente vía para la investigación educativa (Pereira, 2011). En la fase cuantitativa, se parte de cinco dimensiones de análisis, cada una con sus correspondientes nueve preguntas cerradas (tipo Likert). Complementariamente, en el mismo cuestionario se han incluido, junto a las preguntas cerradas, otros tantos apartados de observaciones (preguntas abiertas) para explorar lo que piensan los profesionales de la muestra a partir de sus propias opiniones.

Se incluyen también variables de naturaleza sociodemográfica (género; edad; años de experiencia como profesional de la educación; nivel educativo, titularidad, localización -urbana, rural... - y Estado brasileño de la institución en que trabaja; experiencia educativa en contextos de diversidad cultural y formación previa de carácter intercultural). En la aplicación del cuestionario y en la obtención de los datos, en 2019, se han seguido los procedimientos éticos reconocidos por la comunidad académica.

\section{Muestra}

Se realizó un muestreo no probabilístico y por conveniencia. La muestra total quedó integrada por 237 profesores, 58 hombres (24,5\%) y 179 mujeres (75,5\%). La mayor parte, un $63,1 \%$, tiene 40 años o menos y un $36,9 \%$ tiene 41 años o más. El 68\% lleva 15 años o menos de ejercicio profesional, frente al 32\% que tiene una experiencia profesional de 16 años o más. En lo que se refiere a la etapa educativa en que se trabaja, si bien algunos docentes combinan su actividad profesional en diferentes niveles (un 22,9\%), principalmente de las enseñanzas preuniversitarias, la mayor parte trabaja en "Ensino Fundamental", de carácter obligatorio (para escolares entre 6 y 14 años, aproximadamente). Por orden decreciente, los porcentajes de los niveles educativos en que trabajan los profesores, sin obviar que algunos trabajan en varias etapas, son: Ensino Fundamental (41,6\%), Educação Universitária (24,2\%), Educação Infantil (20,8\%), Ensino Médio (14,7\%), Educação Social/Educação Não-formal (7\%) y Formação Profissional (6,5\%). El 83\% del profesorado consultado trabaja en instituciones públicas, el 10\% en instituciones privadas y el $7 \%$ en ambos tipos de instituciones.

La ubicación del centro de trabajo de los profesores participantes es la siguiente: en la capital el $25 \%$, en la zona metropolitana de la capital el $15,7 \%$, en el espacio rural de la capital el $0,8 \%$, en una ciudad del interior 
del Estado el 49,2\%, y en una zona rural del interior del Estado el 9,3\%. Los Estados brasileños en que trabajan los profesores son: Rio Grande do Norte (79,2\%), Pará (11,7\%), Paraíba (4,6\%), Maranhão (3\%), Bahia (1,5\%). Salvo Pará que pertenece al Norte de Brasil, en la región amazónica, los demás son Estados del Nordeste.

\section{Análisis de la información}

Se utilizó el paquete estadístico SPSS para Windows (versión 25) y se hicieron distintos análisis: análisis exploratorio de los datos, análisis relacional, realización de tablas de variables agrupadas en función de la dimensión y variable sociodemográfica y, por último, interpretación de datos y discusión.

\section{Resultados de carácter cuantitativo}

Se ofrecen las medias y otros estadísticos descriptivos de la muestra en cada una de las dimensiones analizadas (Tabla 1).

Tabla 1. Estadísticos descriptivos de la muestra en las cinco dimensiones analizadas

\begin{tabular}{llcccccc}
\hline & & $\mathrm{N}$ & Media & Mediana & Desviación & Mínimo & Máximo \\
\hline Concepción & Hombre & 57 & 4,21 & 4,33 & 0,47 & 2,78 & 5,00 \\
sobre la diversidad & Mujer & 177 & 4,16 & 4,22 & 0,47 & 2,33 & 5,00 \\
cultural & Total & 234 & 4,17 & 4,22 & 0,47 & 2,33 & 5,00 \\
Práctica & Hombre & 57 & 4,08 & 4,22 & 0,59 & 2,44 & 5,00 \\
de la diversidad & Mujer & 175 & 4,12 & 4,22 & 0,49 & 2,33 & 5,00 \\
cultural & Total & 232 & 4,11 & 4,22 & 0,51 & 2,33 & 5,00 \\
Formación & Hombre & 57 & 3,62 & 3,66 & 0,70 & 2,22 & 5,00 \\
intercultural del & Mujer & 175 & 3,55 & 3,66 & 0,66 & 1,00 & 4,89 \\
profesorado & Total & 232 & 3,57 & 3,66 & 0,67 & 1,00 & 5,00 \\
Actuación & Hombre & 57 & 3,23 & 3,22 & 0,68 & 1,89 & 4,56 \\
intercultural & Mujer & 173 & 3,39 & 3,44 & 0,67 & 1,44 & 5,00 \\
institucional & Total & 230 & 3,35 & 3,44 & 0,68 & 1,44 & 5,00 \\
Currículum & Hombre & 57 & 3,81 & 3,66 & 0,82 & 2,22 & 5,00 \\
intercultural & Mujer & 173 & 3,85 & 4,00 & 0,73 & 1,67 & 5,00 \\
& Total & 230 & 3,84 & 4,00 & 0,75 & 1,67 & 5,00 \\
\hline
\end{tabular}

Fuente: elaboración propia. 
La observación de las medias totales de cada una de las cinco dimensiones (Tabla 1), todas integradas por 9 ítems, y de las medias concretas obtenidas a partir de las respuestas de los profesores a cada una de las 45 preguntas del cuestionario permite afirmar lo siguiente:

En relación a la "Concepción de los profesionales de la educación sobre la diversidad cultural en la educación" (CO), con media 4,17, los profesores dicen conocer qué es la diversidad cultural, hacia la que manifiestan una actitud positiva, que se extiende al abordaje pedagógico de la misma. Se considera que el equipo educativo en su conjunto, con necesaria implicación de la dirección institucional, debe encargarse de esa atención en cuanto fundamento de la educación actual.

La segunda dimensión: "Práctica de los profesionales de la educación en el ámbito de la diversidad cultural" (PR), cuya media es 4,11, pone de manifiesto que el profesorado, en su conjunto, es sensible a la diversidad cultural y a su fomento en las aulas, hasta el punto de que promueve el respeto entre los alumnos, agrupaciones oportunas, según las actividades, y cultiva la colaboración docente para construir un ambiente intercultural.

En la tercera dimensión: "Formación de los profesionales de la educación en el campo de la diversidad cultural" (FO), con media 3,57, se advierte que, si bien el profesorado es consciente de las dificultades que los alumnos de otros países o pertenecientes a culturas minoritarias pueden tener, cuando se pregunta sobre la formación recibida y los cursos realizados para mejorar la competencia intercultural la media de las respuestas se sitúa en un lugar central $(3,06)$. La preparación intercultural es para muchos docentes responsabilidad de la institución educativa en su conjunto, no únicamente del profesor. Puntuación media $(2,99)$ es la que se obtiene también cuando los profesores responden sobre la preparación intercultural atribuida al equipo gestor del centro educativo.

La cuarta dimensión: "Percepción de los profesionales de la educación sobre la actuación del centro/asociación en el campo de la diversidad cultural" (Al), cuya media es 3,35, permite advertir que los profesores participantes piensan, en general, que las instituciones educativas en que trabajan valoran la diversidad cultural, la presencia de alumnos de distintas culturas y tratan de desarrollar el potencial de los educandos, cualesquiera que sean sus características culturales. Los docentes consideran que sus respectivas ins- 
tituciones son inclusivas. Pese al expresado carácter institucional inclusivo, se obtienen puntuaciones medias escasamente diferenciadas en lo que se refiere a fomentar la participación de las familias en la organización y en la implementación de actividades interculturales.

La última dimensión es: "El currículo/plan educativo de actuación en el ámbito de la diversidad cultural" (CU), cuya media es 3,84. Las puntuaciones medias más elevadas se alcanzan cuando se responde sobre la realización de ajustes en el currículum para tener en cuenta las necesidades de los alumnos, sobre el fomento de la inclusión y el empoderamiento de todos los alumnos, así como sobre la contemplación de la realidad cultural institucional en las actividades educativas. Las medias, en cambio, revelan cierta indefinición generalizada cuando se pregunta sobre la disponibilidad de recursos necesarios para atender la diversidad cultural.

Además de los análisis descriptivos se realizaron comparaciones mediante la prueba de Kruskall-Wallis para comprobar si las puntuaciones de las cinco dimensiones son significativamente diferentes dependiendo de cada variable sociodemográfica. Al respecto, se hallaron diferencias estadísticamente significativas en las puntuaciones de dos de las dimensiones (PR, FO) analizadas $(p<0.05)$ cuando la variable sociodemográfica era "Experiencia con alumnos de diferentes culturas a lo largo del ejercicio profesional" (Tabla 2). Las puntuaciones medias en ambas dimensiones son más elevadas ("más acuerdo") cuando aumenta la experiencia educativa con alumnos de diversas culturas.

Tabla 2. Estadísticos de pruebaa,b

\begin{tabular}{lccccc}
\hline & $\begin{array}{c}\text { Concepción } \\
\text { sobre la diversidad } \\
\text { cultural }\end{array}$ & $\begin{array}{c}\text { Práctica } \\
\text { de la } \\
\text { diversidad } \\
\text { cultural }\end{array}$ & $\begin{array}{c}\text { Formación } \\
\text { intercultural } \\
\text { del profeso- } \\
\text { rado }\end{array}$ & $\begin{array}{c}\text { Actuación } \\
\text { intercultural } \\
\text { institucional }\end{array}$ & $\begin{array}{c}\text { Currí- } \\
\text { culum } \\
\text { intercul- } \\
\text { tural }\end{array}$ \\
Gallis & 3,905 & 6,076 & 10,911 & 0,612 & 3,024 \\
gl & 2 & 2 & 2 & 2 & 2 \\
Sig. asintótica & 0,142 & 0,048 & 0,004 & 0,736 & 0,220 \\
\hline
\end{tabular}

a. Prueba de Kruskal Wallis; b. Variable de agrupación: "Experiencia con alumnos de diferentes culturas a lo largo del ejercicio profesional"

Fuente: elaboración propia. 
A partir de la prueba de Kruskall-Wallis también se encontraron diferencias significativas en las puntuaciones de cuatro de las dimensiones ( $P R, F O, A I$, CU) analizadas ( $p<0.05$ ) cuando la variable sociodemográfica era "Número de actividades de formación intercultural realizadas en los últimos cinco años" (Tabla 3). En general, se obtienen puntuaciones medias más altas ("más acuerdo") cuando aumenta el número de actividades. Y en las mismas cuatro dimensiones las puntuaciones medias más bajas ("más desacuerdo") se obtienen, en conjunto, cuando no se ha realizado "ninguna actividad".

Tabla 3. Estadísticos de pruebaa ${ }^{a, b}$

\begin{tabular}{|c|c|c|c|c|c|}
\hline & $\begin{array}{c}\text { Concepción } \\
\text { sobre la diversidad } \\
\text { cultural }\end{array}$ & $\begin{array}{l}\text { Práctica } \\
\text { de la di- } \\
\text { versidad } \\
\text { cultural }\end{array}$ & $\begin{array}{l}\text { Formación } \\
\text { intercultural } \\
\text { del profeso- } \\
\text { rado }\end{array}$ & $\begin{array}{l}\text { Actuación } \\
\text { intercultural } \\
\text { institucional }\end{array}$ & $\begin{array}{l}\text { Currí- } \\
\text { culum } \\
\text { intercul- } \\
\text { tural }\end{array}$ \\
\hline $\begin{array}{l}\text { H de Kruskal- } \\
\text { Wallis }\end{array}$ & 5,420 & 18,554 & 30,887 & 11,786 & 17,830 \\
\hline gl & 3 & 3 & 3 & 3 & 3 \\
\hline Sig. asintótica & 0,144 & 0,000 & 0,000 & 0,008 & 0,000 \\
\hline
\end{tabular}

\section{Resultados de carácter cualitativo}

Pese a que en ninguna de las dimensiones de análisis la participación de los profesionales supera el $8 \%$, la riqueza advertida en las respuestas anima a recoger en los resúmenes diversos matices.

En lo que se refiere a la "Concepción de los profesionales de la educación sobre la diversidad cultural en la educación" (CO), con participación del 7,9\% de los profesores, hay consenso en afirmar que la diversidad cultural enriquece el proceso educativo porque ensancha la mirada humana y profesional.

En cuanto a la "Práctica de los profesionales de la educación en el ámbito de la diversidad cultural" (PR), con respuestas del 4,5\% del profesorado, se advierte el fomento del respeto de los alumnos/educandos a las personas de distintas culturas en pro de una convivencia democrática y solidaria. Se sirven de debates dirigidos, ejemplos, análisis de casos y reflexiones que contribuyan al cambio de actitud y al respeto hacia la diversidad cultural. 
En la dimensión: "Formación de los profesionales de la educación en el campo de la diversidad cultural" (FO), sobre la que han realizado observaciones aproximadamente el 3'5\% de los profesores, se advierte que la formación intercultural recibida es muy variada. No son pocos los profesionales que advierten carencias formativas en materia de educación intercultural.

En lo que se refiere a la "Percepción de los profesionales de la educación sobre la actuación del centro/asociación en el campo de la diversidad cultural" (Al), dimensión sobre la que hay observaciones de cerca de un 3,5\% del profesorado, las respuestas revelan que, en general, no se fomenta la participación de las familias en la educación intercultural, ya sea porque se trata de centros universitarios, ya porque no forma parte de la política institucional.

A la última dimensión: "El currículo/plan educativo de actuación en el ámbito de la diversidad cultural" (CU), han respondido aproximadamente el 3,5\% de los profesores de la muestra. Se refleja en las observaciones que en la planificación pedagógica escolar se despliega una perspectiva cultural plural que, de forma desigual, se materializa en la actuación educativa en el aula y en las actividades de carácter interdisciplinar.

\section{Discusión de resultados y conclusiones}

Los educadores manifiestan una actitud positiva hacia la diversidad cultural, que debe atenderse por todo el equipo pedagógico e implicar a la comunidad educativa en su conjunto. Un planteamiento inclusivo así es fundamental en la sociedad y en las instituciones educativas (centros escolares, asociaciones socioeducativas, etc.). Se trataría de una educación que, como afirman Araújo y Gomes (2020), tenga como base la negociación cultural impulsora de una sociedad plural y neutralizadora de los presupuestos culturales hegemónicos.

En la práctica, algunos estudios, como el realizado por Alencar (2014), ponen de manifiesto que en ocasiones algunas de las dificultades encontradas al trabajar en contextos de diversidad cultural se superan gracias precisamente a la actitud favorable del profesorado hacia los procedimientos teóricometodológicos correspondientes a la pedagogía intercultural, así como por la significativa disposición hacia el aprendizaje construido que se deriva de 
la propia actuación profesional. Con todo, es conveniente insistir en que la política educativa ha de dar cobertura legislativa, material y pedagógica a las actuaciones de atención a la diversidad en función de la especificidad de los centros. No puede recaer la mayor parte de la responsabilidad sobre el profesorado, sino que ha de implicarse toda la comunidad educativa. En esta labor coordinada es fundamental la incentivación de los profesionales a través de medidas orientadas a mejorar sus condiciones de trabajo, así como su formación inicial y continuada en materia de educación intercultural.

Es necesario trabajar las competencias interculturales en la formación del profesorado, siquiera sea por la complejidad social y por los nuevos planteamientos pedagógicos (Vila, Cortés y Martín, 2017). La formación de educadores en el campo de la diversidad cultural representa un proyecto deliberado de preparación sensible a las variadas funciones que los profesionales han de realizar, así como a los desafíos que les aguardan en una sociedad plural. Se precisa una formación intercultural que permita prevenir los discursos excluyentes y homogeneizadores (Pansini y Nenevé, 2008). No deja de ser curioso que en gran medida los profesionales de la educación sigan recibiendo una formación que les capacita sobre todo para trabajar en contextos monoculturales. Es urgente, por tanto, una formación intercultural que se proyecte directamente sobre los educandos y de la que dependerá significativamente la calidad de la educación y la convivencia en un planeta que se mundializa.

Se constata en la investigación que el equipo directivo, por supuesto, con un verdadero liderazgo inclusivo, es fundamental en la atención a la diversidad, pero a veces su labor es insuficiente tanto en lo que se refiere a la planificación y al diseño de la intervención educativa intercultural como al fomento de la implicación de toda la comunidad educativa. Al respecto, en la línea de lo afirmado por Pereira y Aparecida (2020), es capital revisar la propia formación y la función de los gestores educativos, que debieran ayudar activamente a los docentes en su diario quehacer. Además, es necesario contar con más recursos materiales y más actividades específicas de naturaleza intercultural tanto de puertas hacia adentro como hacia afuera. En sintonía con Leiva (2012), desde una óptica holística, la construcción de la interculturalidad requiere aunar esfuerzos y potenciar los vínculos socioculturales de y entre las instituciones educativas, así como con la sociedad, con el fin de que las familias, el alumnado, el profesorado y los directivos tejan redes de significado compartido. 
Muchos profesionales de la educación reconocen la necesidad de tener formación intercultural y se muestran sensibles a la diversidad cultural y a su fomento, pero la práctica intercultural sistemática todavía es insuficiente en un significativo número de instituciones educativas. Metodológicamente, los profesionales más sensibilizados se sirven de estrategias como: debates dirigidos, ejemplos, análisis de casos, discusión de textos, proyección de películas, conferencias externas, narraciones, testimonios, senderos ecopedagógicos y actividades de campo, música, etc., y se utilizan, cuando las hay, las TICs, en las que, además, es preciso mejorar su manejo educativo en contextos de diversidad e interconexión.

Un problema expresado por algún educador tiene que ver con la sobrecarga laboral, que supone incluso la asunción de funciones propias de la Administración o de las familias. El exceso de tareas, a menudo en condiciones particularmente precarias, se complica con la frecuente necesidad de completar el salario con el trabajo en distintos centros educativos, a los que en ocasiones se llega después de tomar varios ómnibus. En circunstancias así no siempre es fácil revisar la actuación intercultural. En el estudio firmado por Sánchez (2018) se constata, por ejemplo, la intensificación del trabajo docente, que rebasa la jornada laboral oficial, en Argentina, Brasil y México, algo que seguramente es extrapolable a otros países de la región y que, entre otras consecuencias, se deja sentir en la reducción de la planificación pedagógica.

Al analizar las condiciones materiales en que se realiza la labor educativa en las escuelas públicas de niveles preuniversitarios en Brasil, sobre todo en los Estados del Norte y Nordeste, más pobres y en los que se centra nuestra investigación, Silva y Souza (2014) destacan, pese a los avances de las últimas décadas, la falta de infraestruturas adecuadas, de equipamentos y de materiales didácticos para el profesorado y el alumnado, lo cual ensombrece el panorama de la educación, también en el ámbito de la diversidad cultural.

La formación intercultural de los educadores debe mejorarse. Actualmente, esta preparación, inicial y continua, es muy variada y precisa más apoyo institucional. Se trata de una capacitación en la que tienen que involucrarse activamente los directivos, ellos mismos con necesidades formativas. Los roles que se asumen por los equipos de gestión suelen ser demasiado burocráticos y con arreglo a un planteamiento que parece responder según los 
casos, como sostiene Díez (2014) a partir de su estudio con directivos, a un modelo multicultural o compensatorio, porque, en general, se considera la diversidad cultural más como un problema que como una oportunidad. En el caso concreto de la gestión escolar en Brasil, Vieira y Vidal (2015) apuntan en su investigación que, pese al principio democrático orientador definido legislativamente, su implementación en el sistema público de enseñanza queda asociado a la emergencia de un nuevo modelo de gestión escolar, sobre todo de carácter gerencialista, eficientista, algo con lo que coincide también Parente (2017). Análogamente, Cabral y Araújo (2011) en una investigación sobre gestión escolar en instituciones de enseñanza media en Rio Grande do Norte sostienen que se debaten entre la gestión democrática y la gerencial, con poca cultura escolar de trabajo colectivo, lo que se traduce en escasa implicación de los actores en la vida de los centros educativos.

La investigación realizada nos lleva a afirmar que en aras de la interculturalidad institucional ha de potenciarse más la participación de las familias y de toda la comunidad educativa, al tiempo que se incorporan planes de acogida en centros que no los poseen, particularmente para los sectores más vulnerables a la exclusión, y se promueve un ambiente congruente, en su doble vertiente psicosocial y material. Si parafraseamos a Peñaherrera y Cobos (2011), debe recordarse que el fundamento de la educación intercultural no se localiza únicamente en el respeto al derecho a ser diferente sino en la valoración explícita de la diversidad, lo que se traduce en un modelo institucional en el que todos sus miembros participan y despliegan un sentido de comunidad.

\section{Agradecimientos}

Esta investigación se realizó en el marco de una beca de movilidad internacional "Salvador de Madariaga" del Ministerio de Educación, Cultura y Deporte (España). Dicha ayuda, que agradezco, me permitió realizar una estancia de investigación de seis meses en la Universidade Federal do Rio Grande do Norte (Brasil), a la que también expreso mi gratitud, particularmente a la Profa Dra. Marlúcia Menezes de Paiva, coordinadora del Grupo investigador receptor. También agradezco al Dr. Pedro L. Cuesta Álvaro, de la Universidad Complutense de Madrid, su valiosa ayuda en el tratamiento estadístico de los datos 


\section{Referencias}

Alencar, J. C. P. M. (2014). Educação intercultural e a formação específica de professores indígenas no ensino superior. Revista Fórum Identidades, 16(16), 79-98. Disponible en http://bit.ly/3bc2B4y

Araújo, G. D. \& Gomes, J. (2020). Educação intercultural e os aspectos da lei nº 11645/2008 história e culturas indígenas. Revista de Educação, Ciência e Cultura, 25(2), 73-85. Disponible en http://bit.ly/37HbbYi

Beserra, B. L. R .\& Lavergne, R. F. (2018). Racismo e Educação no Brasil. Recife: UFPE.

Botler, A. M. H. (2012). Repercussões das políticas multiculturais na educação. Educação \& Sociedade, 33(119), 595-609. Disponible en https://bit.ly/2NAzPCH

Cabral, A. N. \& Araújo, A. M. D. A. (2011). Gestão escolar em instituições de ensino médio: entre a gestão democrática e a gerencial. Educação \& Sociedade, 32(116), 745-770. Disponible en https://bit.ly/3u9D30c

Candau, V. M. (2016). Cotidiano escolar e práticas interculturais. Cadernos de Pesquisa, 46(161), 802-820. Disponible en https://bit.ly/3avsihx

Carvalho, E. J. G. \& Faustino, R. C (2016). Educação para a diversidade cultural: reflexões sobre as influências internacionais na atual política educacional. Revista NUPEM, 8(15), 187-208. Disponible en https://bit.ly/3u79JYx

Cuche, D. (2012). A noção de cultura nas ciências sociais. Bauru: Edusc.

Díez, E. J. (2014). La práctica educativa intercultural en Secundaria. Revista de Educación, 363, 12-34. Disponible en https://bit.ly/3qAAp1C

Ferreira, T. J. \& Silva, M.C. P. (2020). Educação intercultural quilombola: saberes insurgentes em evidencia. Brazilian Journal of Development, 6(10), 75286-75302. Disponible en http://bit.ly/2Nimclf

Freire, P. (2019). Pedagogia da autonomía. Saberes necessários à prática educativa. São Paulo: Paz e Terra.

Igreja, R. L. (2016). Combate al racismo y la discriminación racial en Brasil: legislación y acción institucional. Desacatos, 51, 32-49. Disponible en http://bit.ly/3at1Tkm

Leiva, J. J. (2012). La formación en educación intercultural del profesorado y la comunidad educativa. Revista Electrónica de Investigación y Docencia (REID), 2, 8-31. Disponible en http://bit.ly/3qBrpcl

Lorente, M. (2019). Problemas y limitaciones de la educación en América Latina. Un estudio comparado. Foro de Educación, 17(27), 229-251. Disponible en http://bit.ly/2Zp3aTf

Oliveira, L. F.\& Candau, V. M. (2010). Pedagogia decolonial e educação antirracista e intercultural no Brasil. Educação em Revista, 26(1), 15-40. Disponible en https://bit.ly/3u8Mttk

Oliveira, I. A. (2015). Paulo Freire: gênese da educação intercultural no Brasil. Curitiba: CRV.

Pansini, F.\& Nenevé, M. (2008). Educação multicultural e formação docente. Currículo sem Fronteiras, 8(1), 31-48. Disponible en https://bit.ly/3ucNfFQ

Parente, J. M. (2017). Gestão escolar no contexto gerencialista: o papel do diretor escolar. Roteiro, 42(2), 259-280. Disponible en http://bit.ly/3qABWoo 
Peñaherrera, M. \& Cobos, F. (2011). Inclusión y currículo intercultural. Revista Educación Inclusiva, 4(3), 145-153.

Pereira, C. G. \& Aparecida, S. (2020). Tendências e desafios para a política de formação dos gestores: Uma análise interdisciplinar de educação intercultural no campo. Revista Observatório, 6(1), 1-20. Disponible en http://bit.ly/3pveojD

Pereira, Z. (2011). Los diseños de método mixto en la investigación en educación: una experiencia concreta. Revista Electrónica Educare, 15(1), 15-29. Disponible en https://bit. ly/37orSaL

Sánchez, M. (2018). La intensificación del trabajo docente en tres países latinoamericanos. Voces y Silencios: Revista Latinoamericana de Educación, 9(1), 4-27. Disponible en https://bit.ly/3dp6u9a

Senna, L. A. G. (2000). La educación en Brasil y sus múltiples supuestos: desafíos de la educación intercultural. Revista de Educación, 321, 187-198. Disponible en https://bit.ly/3pEK3zj

Silva, A. F. \& Souza, A. L. L. (2014). Condições do trabalho escolar: desafios para os sistemas municipais de ensino. Cadernos de pesquisa, 43(150), 772-787. Disponible en https://bit.ly/3bjnnz5

Silva, P. \& Vasconcelos, A. M. (2019). Interculturalidade e educação: uma reflexão sobre as políticas multiculturais de educação. Trayectorias Humanas Trascontinentales, 4, 94-102. Disponible en https://bit.ly/2Zs4RiG

Silva, V. A. \& Rebolo, F. (2017). A educação intercultural e os desafios para a escola e para o profesor. Interações, 18(1), 179-190. Disponible en https://bit.ly/3qJc4qo

Vieira, S. L. \& Vidal, E. M. (2015). Gestão democrática da escola no Brasil: desafios à implementação de um novo modelo. Revista Iberoamericana de Educación, 67, 19-38. https://doi. org/10.35362/rie670203

Vila, E., Cortés, P. \& Martín, V. (2017). La formación del profesorado y la importancia de las competencias interculturales y emocionales desde la perspectiva ética. MODULEMA. Revista Científica sobre Diversidad Cultural, 1, 5-20. Disponible en http://bit.ly/3ppJEk0 